\title{
Action of Pulse Magnetic Field on Molten Metal
}

\author{
Anatoly Fedorovich KOLESNICHENKO, Aleksandr Dmitrievich PODOLTSEV \\ and Irina Nikolaevna KUCHERYAVAYA
}

Institute of Electrodynamics, 56, Prospect Pobedy, Kiev-57, 252680, Ukraine. FAX No. 1-044-446-9494.

(Received on April 11, 1994; accepted in final form on June 24, 1994)

\begin{abstract}
Effect of-pulse magnetic field on two-phase zone of crystallizing metal has been investigated by methods of mathematical modelling in reference to vertical continuous casting machine. On the first step of the investigation electromagnetic problem for penetration of pulse magnetic field of cylindrical inductor in depth of liquid metal has been solved and distribution of electromagnetic forces in the melt has been obtained at different time moments. With a view to solve this problem combined method has been proposed. It contains boundary element method for description of field in surrounding air medium with inductor and also finite difference method for solving of the problem in molten metal. On the second step of the investigation with the help of obtained distribution of electrodynamic forces problem for acceleration of the melt has been solved. It is determined the optimal relation for pulse duration (in the case of single pulse), when melt velocity reaches its maximum value. Besides, it is shown that magnetic field may be used with the purpose of effect on crystallization zone through shell of solidified metal.
\end{abstract}

KEY WORDS: pulse magnetic field; molten metal; liquid and solid phase; numerical investigation; boundary element method; finite difference method; electromagnetic force; velocity distribution.

\section{Introduction}

A pulse magnetic field is an effective tool for action on electrically conducting liquids. Application of this field with crystallization of liquid metal provides more high level of power force effect on crystallization zone in comparison with the use of stationary or alternating (harmonic) field, when values of Joulean heat, dissipated in the molten metal, and consumed electric power are the same values. That regime of vibropercussive processing of the molten metal makes it possible to improve quality of ingots by macrocrystallite milling, leveling of physical and chemical properties over all volume, elimination of shrinkage porosity. ${ }^{1-5)}$

Figure 1 schematically shows the process of liquid metal crystallization in magnetic-pulse mould. This process is realized in continuous casting machine. On a level with traditional components the device contains induction coil (inductor), which is connected up to power source of repetitive current pulses and built into the mould or fulfilled as a mould.

Force action of a pulse magnetic field on crystallizing metal manifests itself at least in three phenomena. They may be regarded as the following three processing regimes, for analysis of which different models will be suggested:

(1) It is repetitive effect of comparatively prolonged pulses of magnetic field on the liquid phase (zone 1, Fig. 1) in order to obtain substantial amplitude of velocity pulsations for molten metal in its crystallization area. These velocity pulsations lead to the destruction of growing crystals. Moreover, in consequence of additional stirring cross-section temperature of the melt will be equallized and heat abstraction will be accelerated. In the end, these acts brought to milling of melt structure and acceleration of freezing process:

(2) It is repetitive action of magnetic field pulses on shell of solidified metal (zone 2, Fig. 1) to excite mechanical oscillations in the shell. In this case, for efficient effect pulse duration $\tau$ must be chosen as a value, which satisfies such condition, that $\delta \leq \Delta$, where $\delta=\delta(\tau)$ is a depth of magnetic field skin-layer in shell material, depending upon pulse duration $\tau$, and $\Delta$ is an average

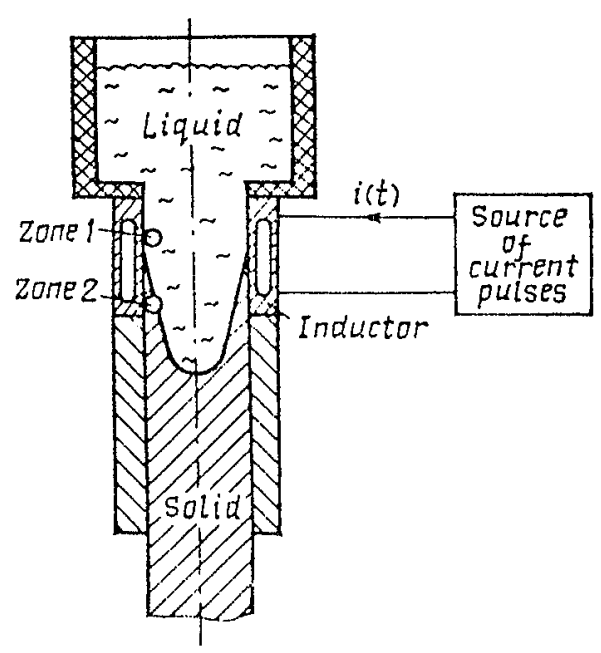

Fig. 1. Schematic representation of crystallization process in magnetic pulse mould. 
shell thickness in interaction area. Besides, the pulse repetition frequency must be in accordance with natural resonant frequency of the shell. Excitations of oscillations in the system "mould-ingot" conduce to more intensive heat abstraction from ingot and to degassing of crystallizing metal. ${ }^{1,6)}$

(3) The repetitive effect of short-term magnetic field pulses with such duration $\tau$, that $\tau<R / v_{s}$ ( $R$ is a typical dimension of ingot cross-section, $v_{s}$ is a sound velocity), leads to the electrodynamic excitation of intensive acoustic oscillations in the melt. Obtained oscillations with definite fixed values of their parameters are favourable ones for improvement of ingot structure. ${ }^{1,3)}$

Each indicated regime is characterized by its own optimal parameters of pulse magnetic field, involving pulse duration, pulse recurrence frequency, peak value of the pulse. In their turn these parameters determine required electrotechnical characteristics of the mould and power pulse source.

The purpose of the research is to fulfil theoretical and numerical investigation of electro- and hydrodynamic processes in molten metal and also to define optimal parameters of magnetic field. The point at issue in the paper is the first above mentioned regime. Consideration of the second and the third regimes ut supra will be executed in the next research work.

\section{Calculation of Electromagnetic Field and Forces}

Electromagnetic interaction under consideration takes place, when magnetic Reynolds number is a small value. In that case, we shall solve the problem by two steps. In the first place, it will be determination of electromagnetic forces in the melt, supposing that velocity of molten metal is $v=0$. And then, on the second step of the investigation we shall find the velocity field according to obtained distribution of electromagentic forces.

As for calculation of time-varying distribution of electromagnetic field and forces in molten metal we shall use combined method: it gives a possibility to apply finite difference method for analysis of processes in the melt and to use boundary element method in space out of inductor. Significant merit of that approach as compared to method of integral equations, using e.g. in Ref. 7) is a simple account of nonlinear characteristics of conductor, namely dependence its electrical conductivity and magnetic permeability on a temperature and also a possibility to consider electromagnetic, thermal and hydrodynamic processes on one and the same space grid.

The calculating model is shown in Fig. 2. It contains round multiturn coil with defined time-variated current $i(t)$ and ingot of cylindrical form with electrical conductivity $\sigma_{2}$ and magnetic permeability $\mu_{2}$. The consideration is built in cylindrical coordinate system $r O z$ for vector potential $\vec{A}$ with only one $\theta$-component. This component of vector potential $\vec{A}$ in region $S_{1}$ out of the ingot is designated as $A_{1}$. And $A_{2}$ designates the $\theta$-component of $\vec{A}$ in ingot's region $S_{2}$. Boundary element method has been used for calculation of electromagnetic field in unrestricted region $S_{1}{ }^{8}$ )

Applying the second Green's formula ${ }^{9)}$ over region $S_{1}$

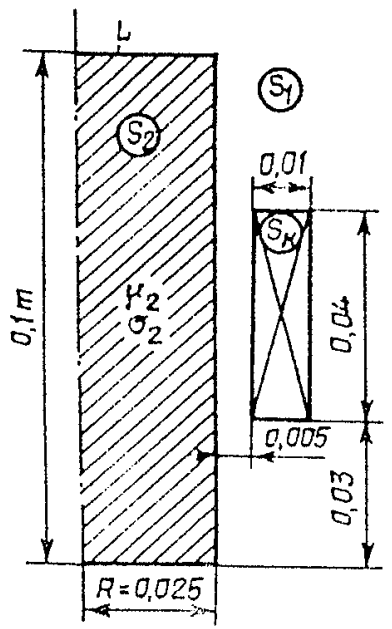

Fig. 2. The calculating model of the system.

and fundamental solution of Laplacian operator in cylindrical coordinate system, designated as $G(Q, M)$, problem in the region $S_{1}$ can be reformulated in form of the next boundary integral expression:

$$
\begin{aligned}
& c_{1}(Q) A_{1}(Q) \\
& =\int_{L}\left[A_{1}(M) \frac{\partial G(Q, M)}{\partial n_{M}}-G(Q, M) \frac{\partial A_{1}(M)}{\partial n_{M}}\right] d L_{M} \\
& +\mu_{0} \iint_{S_{\mathbf{K}}} G(Q, P) j_{c}(P, t) d S_{P} ; \\
& c_{1}(Q)= \begin{cases}1, & Q \in S_{1}, \\
1 / 2, & Q \in L, \\
0, & Q \in S_{2} .\end{cases}
\end{aligned}
$$

Here vector potential $A_{1}(Q)$ is determined in point $Q$; $M, P$ are the running integration points; $n_{M}$ is a normal to contour $L$ in point $M$ directed from region $S_{2}$ to region $S_{1} ; j_{c}$ is a current density in the coil. It is a matter of passing interest, $c_{1}(Q)=1 / 2$ in Eq. (2) merely on smooth section of bound (limit) $L .^{9)}$

Fundamental solution $G(Q, M)$ satisfies the equation:

$$
\nabla^{2} G-\frac{G}{r^{2}}=-\delta\left(r_{Q}-r_{M}, z_{Q}-z_{M}\right)
$$

( $\delta$ is a Dirac delta function) and it is defined from the following expression ${ }^{10)}$ :

$$
\begin{array}{r}
G(Q, M)=\frac{1}{2 \pi} \sqrt{\frac{r_{M}}{r_{Q}}}\left[\left(\frac{2}{k}-k\right) K(k)-\frac{2}{k} E(k)\right] ; \\
k^{2}=\frac{4 r_{Q} r_{M}}{\left(r_{Q}+r_{M}\right)^{2}+\left(z_{Q}-z_{M}\right)^{2}}, \ldots \ldots \ldots \ldots \ldots \ldots \ldots
\end{array}
$$

where $K(k), E(k)$ are complete elliptic integrals of the first and the second kind respectively. If value of $A_{1}(Q)$ on the bound $L$ is the known quantity, then expression (1) may be considered as integral equation relatively variable $\frac{\partial A_{1}}{\partial n_{M}}$ and, on the contrary, if value of $\frac{\partial A_{1}}{\partial n_{M}}$ is the known, then expression (1) is integral equation relatively the unknown $A_{1}$.

To digitizate Eq. (1) let us divide bound $L$ on $N$ 
boundary elements and moreover locate angular point in joints of the elements. In simple case of the use of boundary element method nodes are accommodated in middle of the elements and it is assumed that magnitudes $A_{1}^{j}$ and $\frac{\partial A_{1}^{j}}{\partial n_{M}}$ are constant values within the each element under the number $j$. After combination of point $Q$ with $j$-th angle on smooth section of the bound $L$ $\left(c_{1}(Q)=1 / 2\right)$ and substitution of integrals by sums Eq. (1) may be written in the following matrix form:

$$
[H]\left\{A_{1}\right\}=\left[Q_{n}\right]\left\{\frac{\partial A_{1}}{\partial n}\right\}+\left\{A_{c}\right\},
$$

where $\left\{A_{1}\right\},\left\{\frac{\partial A_{1}}{\partial n}\right\}^{\circ}$ are column vectors with components $A_{1}^{j}$ and $\frac{\partial A_{1}^{j}}{\partial n_{M}}$ respectively; in addition, $[H]$ and $\left[Q_{n}\right]$ are square $N \times N$ matrices with the components:

$$
\begin{aligned}
& H_{i j}=\left\{\begin{array}{l}
-\int_{L_{j}} \frac{\partial G_{1}(Q, M)}{\partial n_{M}} d L_{M}, \quad i \neq j ; \\
\pi r_{i}, \quad i=j ;
\end{array}\right. \\
& Q_{i j}=\left\{\begin{array}{l}
-\int_{L_{j}} G_{1}(Q, M) d L_{M}, \quad i \neq j ; \\
-r_{i} l_{i} \ln \left(\frac{16 r_{i}}{l_{i}}-1\right), \quad i=j,
\end{array}\right.
\end{aligned}
$$

where $r_{i}$ means node $r$-coordinate of the $i$-th boundary element, $l_{i}$ is a length of this boundary element, $G_{1}(Q, M)=2 \pi r_{Q} G(Q, M)$. Integrals in Eqs. (7) and (8) has been calculated with the help of three-point Gauss scheme. Expression for $\frac{\partial G_{1}(Q, M)}{\partial n_{M}}$ has been obtained with the use of results of Ref. 11).

Components of column-vector $\left\{A_{c}\right\}$ in Eq. (6) are vector potential values in node of each boundary element, depending on coil current. These values have been defined by numerical calculation of integral in Eq. (1), taken through a surface $S_{K}$. Obtained matrix expression (6) will be considered further as a boundary condition attached to solution of inner problem in molten metal.

Distribution of vector potential in molten metal satisfies the following differential equation:

$$
\sigma_{2} \frac{\partial A_{2}}{\partial t}=\frac{\partial}{\partial z} \frac{1}{\mu_{2}} \frac{\partial A_{2}}{\partial z}+\frac{\partial}{\partial r} \frac{1}{\mu_{2} r} \frac{\partial\left(r A_{2}\right)}{\partial r}
$$

under the given initial and boundary conditions:

$$
\begin{gathered}
\left.A_{2}\right|_{t=0}=0 ; \\
A_{1}=A_{2} ; \quad\left[\frac{1}{\mu_{0}} \operatorname{rot} \vec{A}_{1}-\frac{1}{\mu_{2}} \operatorname{rot} \vec{A}_{2}, \vec{n}\right]=0 \\
\text { on the bound } L .
\end{gathered}
$$

Finite difference method has been used to solve this problem. Calculating region $S_{2}$ has been covered by space grid and nodal points of boundary elements have been matched with boundary nodes of the grid. Over and above, grid of magnitudes $t$, equally spaced on step value
$\Delta t$, has been introduced in time segment $[0, T]$. Every segment $\left[t_{k-1}, t_{k}\right]$ has been divided into two equal intervals: $t_{k-1} \leqslant t<t_{k-1 / 2}, t_{k-1 / 2} \leqslant t<t_{k}$. Then the following scheme written on the basis of locally one-dimensional difference method ${ }^{12)}$ is a solution of Eq. (9):

$$
\begin{aligned}
& \sigma_{2} \frac{A_{2}^{k-1 / 2}-A_{2}^{k-1}}{\Delta t}=\Lambda_{r} A_{2}^{k-1 / 2}, \\
& \sigma_{2} \frac{A_{2}^{k}-A_{2}^{k-1 / 2}}{\Delta t}=A_{z} A_{2}^{k}, \\
& \sigma_{2} \frac{A_{2}^{k+1 / 2}-A_{2}^{k}}{\Delta t}=A_{z} A_{2}^{k+1 / 2}, \\
& \sigma_{2} \frac{A_{2}^{k+1}-A_{2}^{k+1 / 2}}{\Delta t}=A_{r} A_{2}^{k+1}
\end{aligned}
$$

Here $\Lambda_{r}, \Lambda_{z}$ are finite difference operators corresponding to differential operators in Eq. (9), form of which is given in Ref. 12).

The scheme (10) possesses by absolute stability and has the second order of accuracy, that is to say, deviation of its solution from exact solution is no more than some value $C\left(\Delta t^{2}+h_{r}^{2}+h_{z}^{2}\right)\left(h_{r}, h_{z}\right.$ are steps of the space grid).

Linear algebraic system of Eq. (10) has been solved consecutively on the basis of the simplest formulae of scalaral pass. When electrophysical characteristics is nonlinear, method of simple iteration has been used. Conjugacy of solution of Eq. (9) with boundary expression (6), taking into account Eq. (10), has been fulfilled by iterative process. So, at prescribed values of $\left\{\frac{\partial A_{1}}{\partial n}\right\}$ on molten metal surface values of $\left\{A_{2}\right\}$ obtained from determination of Eq. (9) have been compared with vector potential values from expression (6). When a large divergence has taken place on a certain step of the iterative process, new values of $\left\{\frac{\partial A_{1}}{\partial n}\right\}$ have been determined and the process has repeated-self until coincidence of vector potential values on the bound with definite accuracy.

After calculation of vector potential, value of electromagnetic force per unit volume in molten metal has been obtained in the following manner:

$$
\begin{aligned}
& f_{r}=j_{\theta} B_{z}=-\sigma_{2} \frac{\partial A_{2}}{\partial t} \frac{1}{r} \frac{\partial\left(r A_{2}\right)}{\partial r}, \\
& f_{z}=j_{\theta} B_{r}=\sigma_{2} \frac{\partial A_{2}}{\partial t} \frac{\partial A_{2}}{\partial z} .
\end{aligned}
$$

Further, on the second step of problem solving distribution of velocity field in the molten metal in active operating zone of inductor has been determined on the basis of found force components.

\section{Modelling of Liquid Metal Motion}

The examination of velocity motion in molten metal has been carried out in case of single pulse of magnetic field, supposing that sizable deceleration of the melt (as 
a result of action of viscosity force) takes place in time intervals between two neighbourring pulses. Under these conditions pulse repetition frequency is no too large and accumulation of velocity from each current pulse to the following pulse does not take place.

Molten metal motion under the action of short-term acted electromagnetic forces is described in virtue of Navier-Stokes equation for viscous incompressible liquid and equation of continuity:

$$
\left\{\begin{array}{l}
\frac{\partial \vec{v}}{\partial t}+(\vec{v} \cdot \nabla) \vec{v}=-\frac{1}{\rho} \nabla P+v \nabla^{2} \vec{v}+\frac{\vec{f}}{\rho}, \\
\nabla \cdot \vec{v}=0,
\end{array}\right.
$$

where $\vec{v}$ is a velocity vector for melt, $P$ is a pressure, $\rho, v$ are density of liquid metal and kinematic viscosity respectively, $\vec{f}$ is a vector of electromagnetic forces. In the first equation of the system gravitational force does not take into account, because in case under considera-

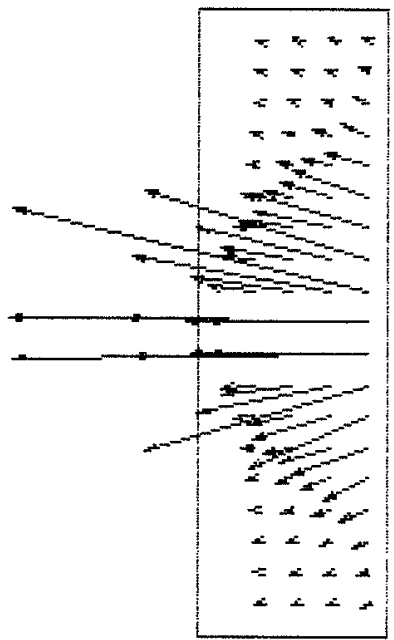

(a)

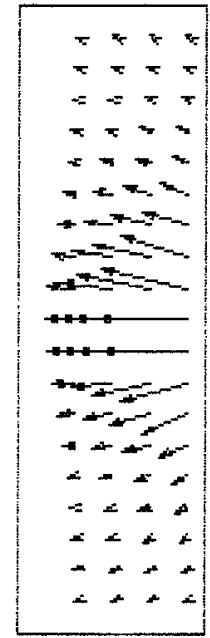

(b)

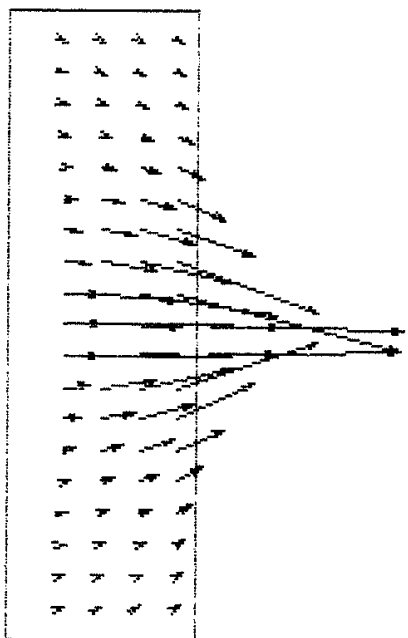

(C)

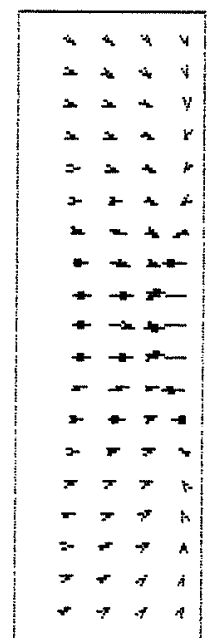

(d)
Fig. 3. Distribution of electromagnetic force in the melt at different time moments: (a) $t=0.25 \cdot 10^{-3} \mathrm{~s}$; (b) $t=0.5 \cdot 10^{-3} \mathrm{~s}$; (c) $t=0.75 \cdot 10^{-3} \mathrm{~s}$; (d) $t=1.0 \cdot 10^{-3} \mathrm{~s}$ $\left(f_{\max }=0.5 \cdot 10^{9} \mathrm{~N} / \mathrm{m}^{3}, \tau=10^{-3} \mathrm{~s}\right)$. tion the condition $\vec{f} \gg \rho \vec{g}$ takes place, where $\vec{g}$ is a free fall acceleration (Fig. 3). Also in that case the deformation of meniscus shape in time of action of single pulse is small owing to a large mass inertia of molten metal volume, which is over zone of electromagnetic interaction.

It may be shown at typical magnitudes of basic variables such as $v \approx 1 \mathrm{~m} / \mathrm{s}, r, z \approx 0.1 \mathrm{~m}, v=10^{-6} \mathrm{~m}^{2} / \mathrm{s}$, pulse duration $\tau=10^{-3} \mathrm{~s}$ (so far as action of single pulse of magnetic field is concerned), in the first equation of Eq. (11) convection term and term, describing of viscosity force, may be neglected. And then Eq. (11) is rewritten in the form:

$$
\left\{\begin{array}{l}
\frac{\partial \vec{v}}{\partial t}=-\frac{\nabla P}{\rho}+\frac{\vec{f}}{\rho}, \\
\nabla \cdot \vec{v}=0 .
\end{array}\right.
$$

After elimination of value of pressure by application of rot-operation to both sides of the first equation of the system (12) and integration with respect to time the following system gets:

$$
\left\{\begin{array}{l}
\operatorname{rot} \vec{v}=\left.\operatorname{rot} \vec{v}\right|_{t=0}+\frac{1}{\rho} \int_{0}^{t} \operatorname{rot} \vec{f} d t, \\
\nabla \cdot \vec{v}=0 .
\end{array}\right.
$$

Further, after introduction of vector potential of velocity $\vec{A}_{v}$, which satisfies the following conditions:

$$
\vec{v}=\operatorname{rot} \vec{A}_{v}, \quad \operatorname{div} \vec{A}_{v}=0,
$$

expressions (13) are rewritten under zero initial conditions as:

$$
\left\{\begin{array}{l}
\nabla^{2} \vec{A}_{v}=\frac{1}{\rho} \int_{0}^{t} \operatorname{rot} \vec{f} d t, \\
\left.\vec{A}_{v}\right|_{L}=0,
\end{array}\right.
$$

where the zero boundary condition for $\vec{A}_{v}$ corresponds to nonleakage of liquid through bound $L$. In case under examination vector $\vec{A}_{v}$ has only one $\theta$-component as a function of variables $A_{v \theta}(r, z, t)$. The problem (Eq. (14)) has been solved by finite difference method with utilization of method of upper relaxation. ${ }^{13)}$ Common space grid has been used for this problem as well as for electromagnetic problem.

It should be noted that given algorithm doesn't describe process of velocity damping after stoppage of electromagnetic force action and also velocity distribution in border layer near the walls of mould. It is necessary to use supplementary and more complicated models with the purpose of consideration of these phenomena.

\section{Computed Results}

Dimensions of investigating system are shown in Fig. 2. Molten steel is considered as material of the liquid metal; its physical data are $\sigma_{2}=0.73 \cdot 10^{6}(\Omega \cdot \mathrm{m})^{-1}$, $\rho=7100 \mathrm{~kg} / \mathrm{m}^{3}$. The number of winding turns of the inductor is $w=1$. Pulse current in the induction-coil is changed as: 


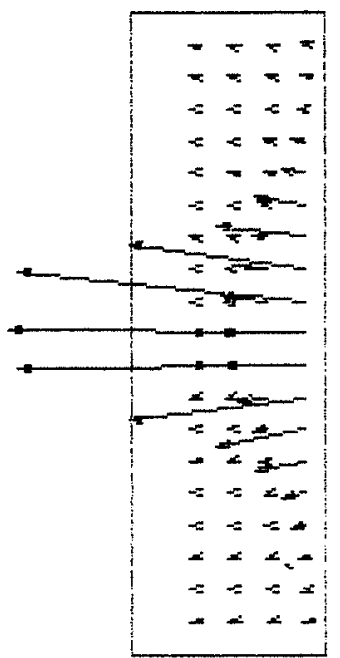

(C)

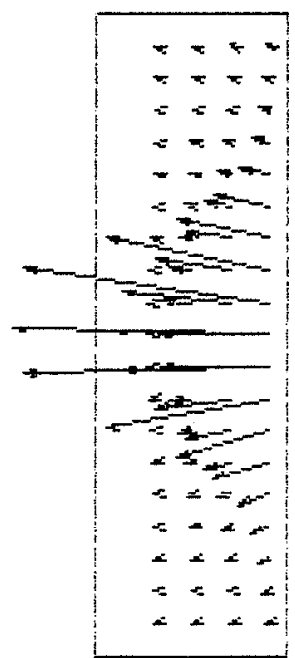

(b)

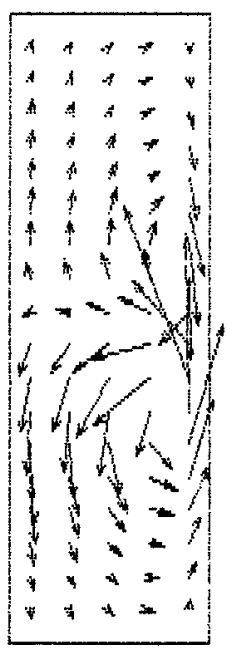

(Cl)

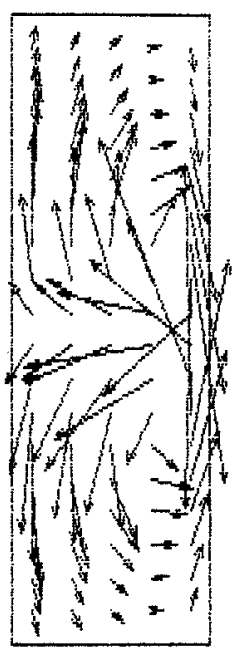

(b)
Fig. 5. Velocity filed at time moments: (a) $t=0.5 \cdot 10^{-4} \mathrm{~s}$; (b) $t=1.0 \cdot 10^{-4} \mathrm{~s}\left(v_{\max }=2.62 \mathrm{~m} / \mathrm{s}, \tau=10^{-4} \mathrm{~s}\right)$.

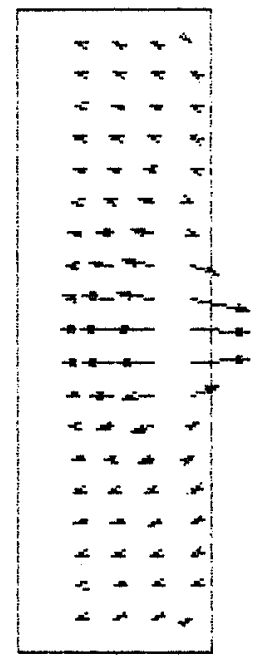

(c)

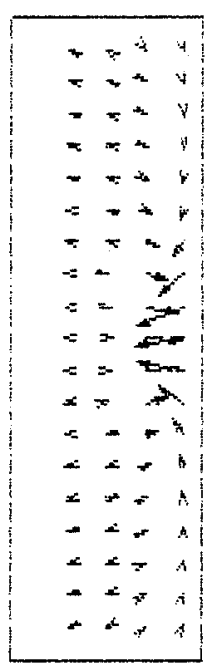

(d)
Fig. 4. Distribution of electromagnetic force in the melt at different time moments: (a) $t=0.25 \cdot 10^{-4} \mathrm{~s}$; (b) $t=0.5 \cdot 10^{-4} \mathrm{~s}$; (c) $t=0.75 \cdot 10^{-4} \mathrm{~s}$; (d) $t=1.0 \cdot 10^{-4} \mathrm{~s}$ $\left(f_{\max }=0.3 \cdot 10^{10} \mathrm{~N} / \mathrm{m}^{3}, \tau=10^{-4} \mathrm{~s}\right)$.

$$
i(t)=\left\{\begin{array}{l}
i_{\max } \sin \pi t / \tau, \quad 0 \leqslant t \leqslant \tau \\
0, \quad t>\tau,
\end{array}\right.
$$

where the current amplitude is $i_{\max }=3.2 \cdot 10^{5} \mathrm{~A}$ and pulse duration $\tau$ is varied within the range $10^{-4} \cdots 10^{-3} \mathrm{~s}$.

Figures 3 and 4 illustrate the computed distributions of electromagnetic force in the liquid metal at different time moments, when pulse duration $\tau=10^{-3}$ and $10^{-4} \mathrm{~s}$, respectively. It is shown maximum magnitude of vector $\vec{f}$ grows, when the pulse duration $\tau$ decreases. At the end of current flow through inductor direction of the vector $\vec{f}$ is changed and the liquid metal is attracted towards inductor. This phenomenon strengthens with increase of pulse duration $\tau$ or, that is to say, with extension of penetration depth of magnetic field in the melt: $\delta=\delta(\tau)=0.57 \sqrt{\tau /\left(\mu_{2} \sigma_{2}\right)}$, where factor 0.57 conforms to chosen form of current pulse in the inductor. ${ }^{14)}$ As a

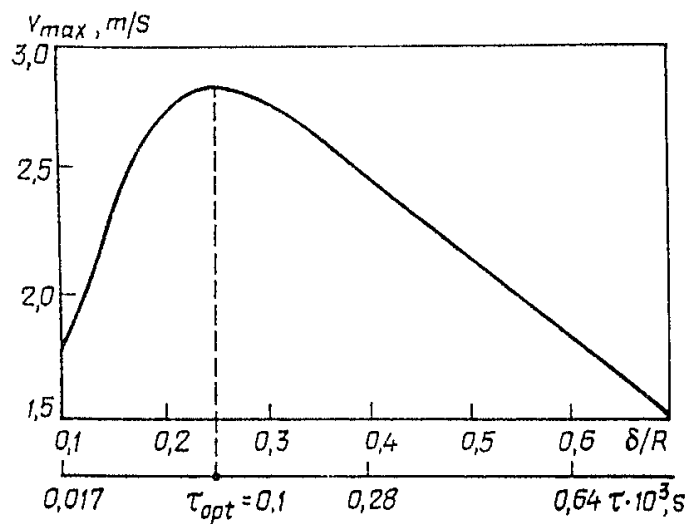

Fig. 6. Influence of pulse duration $\tau$ on maximum value of melt velocity $v_{\max }$

matter of record, the distribution of melt velocity at $\tau=10^{-4} \mathrm{~s}$ is shown in Fig. 5. It has a behaviour of vorticity.

Further, values of pulse duration have been varied to light the influence of $\tau$ on intensity of liquid metal stirring. The influence of the duration $\tau$ on peak value of melt velocity $v_{\max }$ is given in Fig. 6. Here $v_{\max }$ is a maximum value of velocity in cross-section of the melt at time moment $t=\tau$. It stands to reason that the best value of pulse duration $\tau=\tau_{\mathrm{opt}}$ exists and then velocity of liquid metal reaches its peak value. In case under consideration $\tau_{\text {opt }}=10^{-4} \mathrm{~s}$ or corresponding to this pulse duration depth of penetration of magnetic field is $\delta \approx 0,24 R$, where $R$ is a radius of ingot. Although magnitude of vector $\vec{f}$ increases, when $\tau<\tau_{\text {opt }}$, but integral $\int_{0}^{t} \operatorname{rot} \vec{f} d t$ in Eq. (13) decreases, because value of $\tau$ decreases too. On the contrary, if $\tau>\tau_{\text {opt }}$, then reduction of the vector $\vec{f}$ occurs, that is why decrease of integral mentioned above takes place.

Pulse field may be used for the action on crystallization zone, when shell of solidified metal with thickness $\Delta$ already exists. Figure 7 shows the velocity distribution in the melt provided shell thickness in inductor zone doesn't change with direction $O z$. As for electromagnetic 


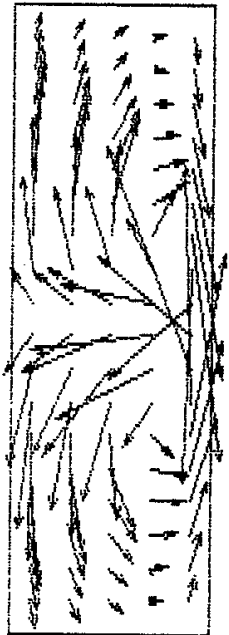

(a)

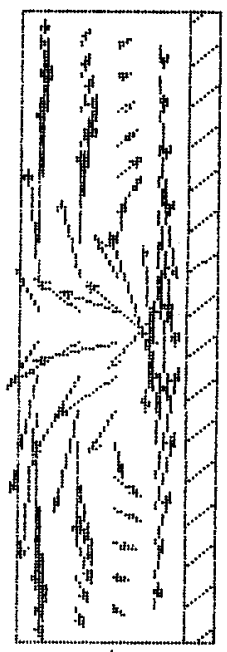

(b)

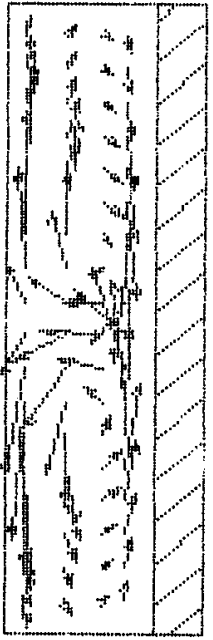

(c)

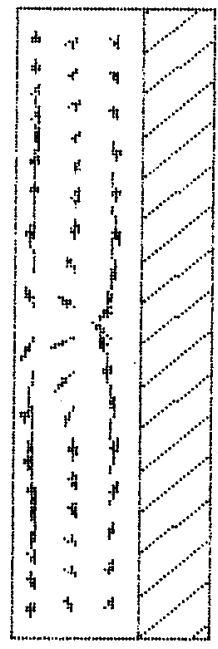

(d)
Fig. 7. Character of velocity distribution $\left.v\right|_{t=\tau}(\tau=0.15$. $10^{-3} \mathrm{~s}$ ) at different values of shell thickness $\Delta$ : (a) $\Delta=0$, $\left(v_{\max }=2.78 \mathrm{~m} / \mathrm{s}\right) ;(\mathrm{b}) \Delta=0.0025 \mathrm{~m}\left(v_{\max }=0.748 \mathrm{~m} / \mathrm{s}\right) ;(\mathrm{c})$ $\Delta=0.005 \mathrm{~m}\left(v_{\max }=0.41 \mathrm{~m} / \mathrm{s}\right) ;(\mathrm{d}) \Delta=0.0075 \mathrm{~m}\left(v_{\max }=\right.$ $0.228 \mathrm{~m} / \mathrm{s}$ )

problem, it was supposed that electrical conductivity of liquid phase is equal to value of the conductivity of solidified shell. It is obvious (see Fig. 7), availablity of the shell leads to considerable decrease of melt velocity. For the choice of the best value $\tau_{\text {opt }}$ Figure 8 illustrates the curves, which are analogous to the curve in Fig. 6, but already for different magnitudes of $\Delta$. It is shown, with increase of shell thickness $\Delta$ optimal value $\tau_{\text {opt }}$ or else $\delta / R$ increases. When $\delta / R \lesssim 0,15$ (that is to say $\delta / \Delta \lesssim 1$ ), force effect on the liquid metal is insignificant in a value.

Thus far liquid metal motion near shell of solidified metal in regard to vertical line of demarcation "liquidsolidified metal" has been discussed. For metals with high thermal conductivity the demarcation line of mediums may be located nearly horizontally. Further,

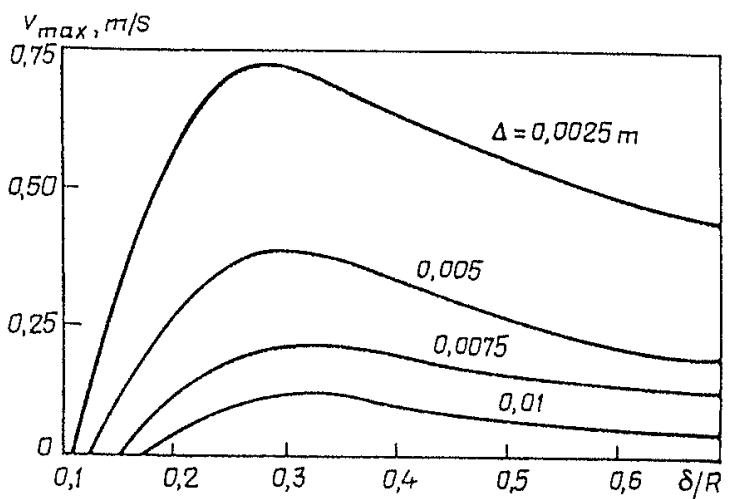

Fig. 8. Influence of skin-layer depth $\delta / R$, depending on pulse duration $\tau\left(\delta(\tau)=0.57 \sqrt{\left.\tau /\left(\mu_{2} \sigma_{2}\right)\right)}\right.$, on maximum value of melt velocity $v_{\max }$ at different magnitudes of shell thickness $\Delta$.

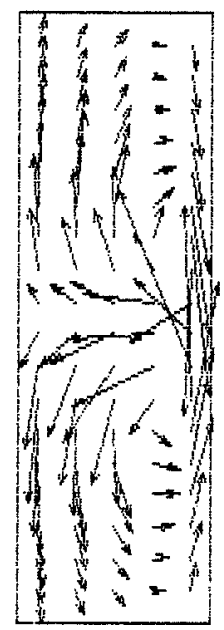

(a)

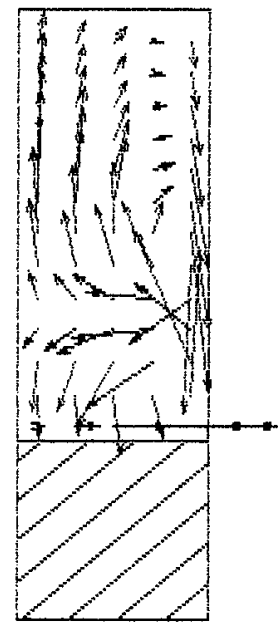

(b)

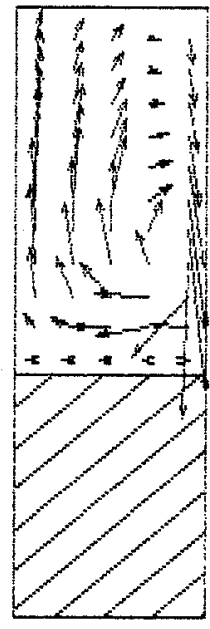

(C)

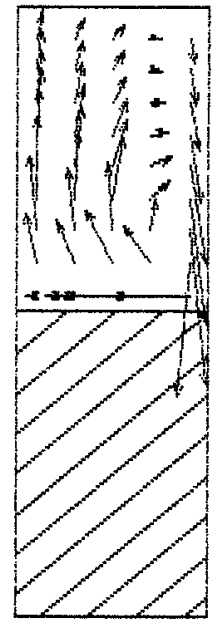

(d)

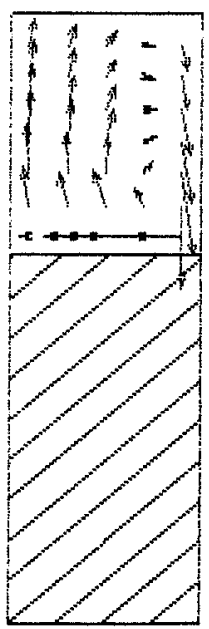

(e)
Fig. 9. Velocity distribution $\left(\left.v\right|_{t=t}, \quad \tau=10^{-3} s\right)$ : (a) $h=0$ $\left(v_{\max }=1.35 \mathrm{~m} / \mathrm{s}\right) ;\left(\right.$ b) $h=0.03 \mathrm{~m}\left(v_{\max }=1.19 \mathrm{~m} / \mathrm{s}\right)$; (c) $h=0.04 \mathrm{~m}\left(v_{\max }=1.13 \mathrm{~m} / \mathrm{s}\right) ;($ d $) h=0.05 \mathrm{~m}\left(v_{\max }=1.07\right.$ $\mathrm{m} / \mathrm{s})$; (e) $h=0.06 \mathrm{~m}\left(v_{\max }=0.57 \mathrm{~m} / \mathrm{s}\right)$.

melt motion near horizontal line is considered at different inductor positions. It was supposed that electrical conductivity of liquid phase is equal to the conductivity 
of solid phase. So, the velocity distribution in the liquid phase is shown in Fig. 9. It is obvious from Fig. 9, if position of medium interface is below horizontal axis of symmetry of the inductor (Figs. 9(b), (c)), then melt motion near this line is directed from centre to lateral wall of the canal. On the contrary, if location of the boundary is above the axis of symmetry, then particles of the liquid carry towards centre (Figs. 9(d), (e)). Thereby, it is possible, for example, to install two inductors and to feed them by current pulses in turns in order to obtain different directions of melt velocity in crystallization area.

\section{Conclusion -}

The numerical model has been developed to analyze the electromagnetic and hydrodynamic processes in molten metal, which is under the action of pulse magnetic field of cylindrical inductor. In the course of theoretical and numerical investigation optimal value of pulse duration in induction coil (when the molten metal accelerates to maximal velocity in time of force action of electromagnetic field) has been determined. The influence of pulse duration as well as the influence of inductor position on motion of liquid metal in the presence of solidified phase have been shown. That solidified phase of molten metal appears as electromagnetic screen for pulse magnetic field. The theoretical results may be used for creation of new technological processes on the basis of non-steady MHD effects.

\section{REFERENCES}

1) G. I. Eskin: Ultrasonic Processing of Melted Aluminium, Metallurgiya, Moscow, (1965).

2) A. Kolesnichenko, A. Podoltsev and B. Jushchenko: Proc. Symp. on Magnetohydrodynamics in Process Metallurgy, The Minerals, Metals \& Materials Society, TMS, Warrendale, Pennsylvania, USA, (1992), 223.

3) M. C. Mascre: Fonderie, 200 (1962), 361.

4) A. E. Mikelson and Z. D. Cherny: Electrodynamic Excitation and Measurement of Oscillations in Metals, Zinatne, Riga, (1979), 151.

5) M. Nakada, Y. Shiohara and M. Flemings: ISIJ Int., 30 (1990), 27.

6) I. G. Saucedo and K. E. Blazek: Proc. of 6th Int. Iron and Steel Congress, ISIJ, Tokyo, (1990), 325.

7) J. Sakane, K. Tunenary, E. Takeuchi, I. Miyoshino and H. Yano: Proc. of 6th Int. Iron and Steel Congress, ISIJ, Tokyo (1990), 400.

8) C. A. Brebbia and S. Walker: Boundary Element Techniques in Engineering, Butterworth \& Co. (Publishers) Ltd., London, UK, (1980).

9) A. N. Tichonov and A. A. Samarskii: Equations of Mathematical Physics, Nauka, Moscow, (1972), 795.

10) L. R. Neiman: Theory of Electromagnetic Field, Cosenergoizdat, Moscow-Leningrad, (1959), 232.

11) O. V. Tozoni: Calculation of Electromagnetic Field by Means of Computer, Technika, Kiev, (1967), 252.

12) A. A. Samarskii: Theory of Difference Schemes, Nauka, Moscow, (1989), 616.

13) L. A. Hageman and D. M. Young: Applied Iterative Methods, Academic Press, New York, USA, (1981).

14) H. Knoepfel: Pulsed High Magnetic Fields, North-Holland Publishing Company, Amsterdam-London, (1970). 